Please do not remove this page

RMIT

UNIVERSITY

\title{
Numerical analysis of neutron moisture probe measurements
}

Li, Jie; SMITH, D; FITYUS, S; SHENG, D

https://researchrepository.rmit.edu.au/esploro/outputs/9921861861101341/filesAndLinks?institution=61RMIT_INST\&index=null

Li, J., SMITH, D., FITYUS, S., \& SHENG, D. (2003). Numerical analysis of neutron moisture probe measurements. International Journal of Geomechanics, 3(1-2), 11-20.

https://doi.org/10.1061/(ASCE)1532-3641(2003)3:1(11

Published Version: https://doi.org/10.1061/(ASCE)1532-3641(2003)3:1(11

Repository homepage: https://researchrepository.rmit.edu.au

(c) 2003 ASCE

Downloaded On 2023/04/26 21:30:26 +1000 
Thank you for downloading this document from the RMIT Research Repository.

The RMIT Research Repository is an open access database showcasing the research outputs of RMIT University researchers.

RMIT Research Repository: http://researchbank.rmit.edu.au/

\section{Citatioon:}

Li, J, Smith, D, Fityus, S and Sheng, D 2003, 'Numerical analysis of neutron moisture probe measurements', International Journal of Geomechanics, vol. 3, no. 12, pp. 11-20.

See this record in the RMIT Research Repository at:

http://researchbank.rmit.edu.au/view/rmit:19620

Version: Accepted Manuscript

Copyright Statement: (c) 2003 ASCE

Link to Published Version:

http://dx.doi.org/10.1061/(ASCE)1532-3641(2003)3:1(11) 
The Numerical Analysis of Neutron Moisture Probe Measurements

Jie Li, David W. Smith, Stephen G. Fityus and Daichao Sheng

Department of Civil, Surveying and Environmental Engineering

The University of Newcastle

Callaghan, NSW 2308, Australia

\section{Correspondence to:}

Dr Jie Li

Department of Civil, Surveying and Environmental Engineering The University of Newcastle

Callaghan, NSW 2308

Australia

Phone: 610249215582

Fax: 610249216991

Email: jli@ mail.newcastle.edu.au 


\title{
The Numerical Analysis of Neutron Moisture Probe Measurements
}

\author{
Jie Li, David W. Smith, Stephen G. Fityus and Daichao Sheng
}

\begin{abstract}
The neutron probe has proven to be an effective means for monitoring long term in situ soil moisture variations. However, it is difficult to experimentally correlate neutron probe data (i.e. neutron counts) with accurate estimates of absolute soil moisture content, particularly for unsaturated clay soils. In this paper, a numerical model based on multigroup neutron diffusion theory is employed to predict the distribution of neutron flux in a neutron probe system. The model discretizes the neutron energy spectrum into seven intervals, with energy-dependent diffusion coefficients and parameters for each energy interval. The finite element method is employed to solve the coupled seven-group neutron diffusion equations. It is demonstrated that the numerical results compare very well with both laboratory experimental results and field measurements. The theoretical approach to neutron probe calibration described herein offers significant time and cost savings over traditional calibration methods, and potentially opens up new applications for neutron probe monitoring.
\end{abstract}

\section{Key Words:}

neutron probe calibration, soil moisture, multigroup neutron diffusion equations, finite element method 


\section{INTRODUCTION}

The neutron-scattering method is widely used in agriculture, forestry, hydrology and civil engineering, for measuring the water content of soil. The main advantages of the neutron method compared to the gravimetric method are: (a) it is non-destructive, (b) it is fast, and (c) repeated measurements can be carried out in situ.

Although the neutron probe has proven to be a convenient and effective means for monitoring long-term in situ soil moisture variations (Schmugge et al, 1980; Silvestri et al, 1991), the experimental correlation of neutron probe data (i.e. neutron counts) with moisture contents to give accurate absolute values of moisture content is a difficult task, particularly for clay soils. Neutron probe counts in moist soils are influenced by the moisture content, soil elemental composition, soil density, and proximity of the probe to the water table and soil surface (Dickey, 1990). Neutron counts are also influenced by the strength of the neutron source, the size and type of the neutron detector, the position of the detector relative to the source, and the size and type of access tube (Stone, 1990; Schmugge et al. 1980). It has been shown that factory calibrations are often inaccurate (see for example Bell and McCulloch, 1969; Rawls and Asmussen, 1973; Vachaud et al., 1977; Carneiro and Jong, 1985; Chanasyk and Naeth 1996). For example, Silvestri et al. (1991) found that the factory calibration curve supplied by the manufacturer was only applicable for sandy soil (with no significant amount of absorbing elements or organic materials), and only for volumetric water contents ranging from 0 to $33 \%$. Calibration is therefore necessary for each type of soil, different types of access tube, and different measuring locations with respect to the soil surface and water table.

Calibration of a neutron probe involves correlating neutron counts with known volumetric water contents of the soil. Two experimental approaches that are commonly employed: laboratory drum calibration, and in situ or field calibration. Laboratory calibrations are made by packing a drum of suitable dimensions with the soil having a range of known moisture contents, installing an access tube as used in the field, and measuring the neutron probe counts. The radius of the drum must be larger than the radius of influence of the neutron probe to prevent neutron leakage. The soil used in laboratory calibrations should have the same elemental composition and bulk density as the soil in the field. However, it is usually difficult to reproduce in a drum the soil fabric found in situ (IAEA, 1970).

Field calibrations are accomplished by correlating the probe readings in an access tube installed in the field, with the estimated volumetric moisture contents of the soil along the tube (or possibly 
immediately adjacent to the tube). These comparisons have to be repeated at different times of the year, so as to sample the soil at different moisture contents. The volumetric moisture contents are usually estimated from gravimetric soil moisture content and soil density. However, it is often difficult to obtain representative soil samples from heterogeneous soil profiles. In addition, the soil moisture content in the field may very rapidly with depth, significantly complicating the interpretation of neutron readings. Detailed descriptions of the laboratory and field calibrations can be found in Greacen (1981) and IAEA (1970).

Even though both laboratory and field calibrations are time consuming and labour-intensive, the results are not always satisfactory, especially for clay soils. Greacen (1981) indicated that early "calibration curves for clay soils" in the literature were "almost invariably wrong". The problem is further complicated for unsaturated expansive clay soils, due to the fact that both soil volume and density change as the in situ soil moisture content changes. Experimental investigations on various types of soil have shown that, in addition to the moisture content, the neutron probe reading depends mainly upon the dry bulk density of the soil (IAEA, 1970).

In some circumstances, it is nearly impossible to obtain an experimental calibration. Morris and Williams (1990) discussed the attempted neutron probe calibration of a fine-grained coal mine tailings first deposited as a slurry, and then sedimented and finally consolidated. They found that it was impractical to directly measure density profiles with depth as the majority of the tailings were too soft to sample. In another example, Elder and Rasmussen (1994) reported that the experimental calibrations were unsuitable for some geologic media, such as unsaturated tuff, due to the difficulty of extracting undisturbed samples for volumetric moisture content measurement.

Because the neutron probes can be so difficult to calibrate by experimental methods, it is desirable to develop an alternative and more reliable calibration method. The purpose of this study is to develop a generalized calibration method from first principles that can be used for all media. The method is based on a numerical model of the neutron probe system and on the elemental composition of the medium in which the neutron probe is used. The theory behind the model is known as 'multigroup neutron diffusion theory' (Isbin, 1963; Zweifel, 1973; Iliffe, 1982; Stacey 2001). The numerical model predicts the count rate for a neutron probe based on dry density, moisture content, and elemental composition of the soil, and the known geometry and size of the neutron detector and source, and strength of the neutron source. 


\section{THEORETICAL BACKGROUND}

\subsection{Physical Phenomena Involved in Neutron Moisture Gauges}

The neutron moisture gauge consists of a probe containing a source of fast (high-energy) neutrons that move radially outward from the source, a thermal neutron detector, and the associated electronic equipment necessary to supply power for the detector and to display the results. A gauge is illustrated in Figure 1. Measurements with depth are made by lowering the probe down an access tube, usually made of either stainless steel or aluminum, to the required depths of measurement. Aluminum is generally the material of choice because it has the low absorption cross-section for slow (thermalized) neutrons. Stainless steel tubes may have to be used if the soil is corrosive.

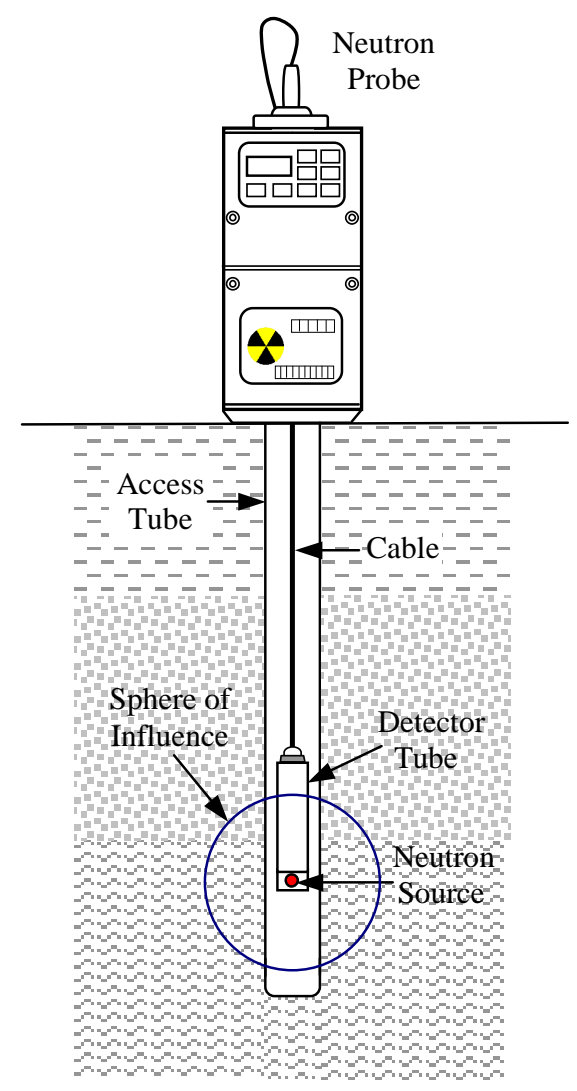

Figure 1. Schematic of a neutron gauge.

The neutron scattering method for measuring soil water content exploits neutron 'thermalization behaviour'. When the neutron probe is in the borehole, fast neutrons emitted by the source collide with the atomic nuclei of the surrounding medium. Each collision between a neutron and a nucleus results in a transfer of energy from the neutron to the nucleus. The average energy 
decrement per collision $(\xi)$ is defined as (Glasstone and Edlund, 1957; Weinberg and Wigner 1958):

$$
\xi=1+\frac{(A-1)^{2}}{2 A} \ln \frac{A-1}{A+1}
$$

where $A$ is the atomic mass of the impacted nucleus.

It can be seen from equation (1) that $\xi$ is dependent only on the atomic mass of the impacted nucleus, and is independent of the initial energy of the neutrons. In other words, after each collision, the neutron always loses the same fraction of its energy. This fraction decreases with increasing atomic mass of the impacted nucleus. Using $\xi$, the average number of collisions required to covert fast neutrons with initial energy of $E_{0}$, say, 2 million electron volts $(\mathrm{MeV})$, to the thermal value of $E=0.025$ electron volts $(\mathrm{eV})$, can be calculated as follows (Gibson, 1980; Glasstone and Edlund, 1957):

$$
\begin{gathered}
\text { Average number of collisions to thermalize } \\
\text { fast neutrons (from } 2 \mathrm{Mev} \text { to } 0.025 \mathrm{eV} \text { ) }
\end{gathered}=\frac{\ln \frac{E_{0}}{E}}{\xi}=\frac{\ln \frac{2 \times 10^{6}}{0.025}}{\xi}=\frac{18.2}{\xi}
$$

The results of a set of these calculations for a number of selected elements are given in Table 1 .

Table 1. Number of collisions required to thermalize a fast neutron for selected elements

\begin{tabular}{llll}
\hline Element & $\begin{array}{l}\text { Atomic } \\
\text { mass }\end{array}$ & $\xi$ & $\begin{array}{l}\text { Number of } \\
\text { collisions }\end{array}$ \\
\hline Hydrogen & 1.008 & 1.000 & 18 \\
Deuterium & 2 & 0.725 & 25 \\
Helium & 4.003 & 0.425 & 43 \\
Lithium & 6.94 & 0.262 & 69 \\
Beryllium & 9.013 & 0.206 & 88 \\
Carbon & 12.011 & 0.158 & 115 \\
Oxygen & 16 & 0.120 & 152 \\
Sodium & 22.991 & 0.085 & 215 \\
Iron & 22.85 & 0.354 & 514 \\
Uranium & 238.07 & 0.00838 & 2172 \\
\hline
\end{tabular}

From the table, it can be seen that hydrogen nuclei have a much greater thermalizing effect on fast neutrons than any other element. Since neutrons and hydrogen atoms have the same mass, fast neutrons are slowed down most effectively by collisions with hydrogen atoms, much like a 
billiard ball hitting a stationary ball of the same size and each moving away with equal speeds (one slowing down and the other speeding up). This is the fundamental reason why a neutron gauge can be employed to detect the proportion of water molecules present in a soil.

\subsection{Neutron Sources}

The radioisotope most commonly employed in moisture gauges is Americium-241. Americium undergoes decay to Neptunium, ejecting an alpha particle in the process. Beryllium, which is incorporated in the source, is bombarded by $\alpha$ particles and converted into carbon and 'fast neutrons' according to the reaction:

$$
{ }_{4}^{9} B_{e}+{ }_{2}^{4} H_{e} \rightarrow{ }_{0}^{1} n+{ }_{6}^{12} C
$$

Most neutron probes also emit a low level of $\gamma$ radiation. $\gamma$ rays may arise from the radioisotope itself or from ${ }_{6}^{12} C$ left in the exited state after conversion from beryllium (IAEA, 1970). At present, the most widely used neutron source is ${ }^{241} \mathrm{Am}-\mathrm{Be}$ because it produces a much lower level of $\gamma$ radiation than a ${ }^{226} \mathrm{Ra}-\mathrm{Be}$ source, and so needs very little shielding. With a half life of approximately 460 years, the ${ }^{241} \mathrm{Am}-\mathrm{Be}$ source can be permanently sealed in a shielded container and maintain an effectively constant neutron production rate for many years.

\subsection{The Neutron Energy Spectrum}

The fast neutrons from the radioactive source in a neutron probe possess a range of velocities and energies. For example, fast neutrons emitted from an Americium-241-Beryllium source have an initial energy of approximately 4.5 MeV. As the fast neutrons diffuse in the soil medium, they are slowed down and lose energy, mainly by elastic collisions with hydrogen nuclei (and to a lesser extent by absorption), and finally become 'thermalized.' Thermalization is the process whereby neutrons are slowed to the point where further collisions with hydrogen and other materials do not continue to slow the neutrons further (i.e. they are just as likely to gain energy as lose energy in collisions once thermalized).

It is important to note that neutron transport involves a spectrum of energy levels, scattering collisions and absorption reactions (see next section). Fast neutrons are defined here as those having kinetic energies in excess of $2 \mathrm{eV}$, while slow neutrons are defined as those with kinetic energy below $2 \mathrm{eV}$. It should be noted that the slow neutrons may be further divided into two 
groups: 'thermal neutrons' having energies from 0 to $0.5 \mathrm{eV}$, and so-called 'epithermal neutrons' with energies between $0.5 \mathrm{eV}$ to $2.0 \mathrm{eV}$ (IAEA, 1970).

\subsection{Neutron Interactions and Cross Sections}

Neutron interactions with the surrounding material can be classified as either absorption reactions or scattering interactions. Absorption is the process where a neutron enters a nucleus, thereby forming a new isotope in an excited state, which usually rapidly relaxes by emitting gamma radiation. Absorption reactions are strongly dependent on the neutron energy level. The absorption of fast neutrons can usually be neglected in ordinary soils since absorption rapidly decreases for energies above the thermal range.

In scattering interactions, the kinetic energy of a neutron is partially or completely transferred to the impacted nuclei in successive collisions through elastic or inelastic scattering. Reactions due to elastic scattering are by far the dominant mode of interaction of fast neutrons in soils (IAEA, 1970).

Neutron interactions with atomic nuclei (whether scattering or absorption), can be quantitatively described through the use of the concept of nuclear cross sections. A cross section is defined as the probability of occurrence of a given type of nuclear interaction. The experimental determination of cross sections is based on the attenuation of a collimated neutron beam passing through a slab of material of finite thickness. Defining $d$ as the distance through the slab in the direction of the beam, the number of neutrons varies with distance through the slab according to,

$$
I=I_{0} e^{-\Sigma d}
$$

where $I_{0}$ is the initial neutron intensity, $I$ the neutron intensity at distance $d$, and $\Sigma$ is called the 'macroscopic cross section.' The units of $\Sigma$ are inverse length (usually $\mathrm{cm}^{-1}$ ), because the exponent $(\Sigma d)$ has no dimension. The 'microscopic cross section' denoted $(\sigma)$ is obtained by dividing $\Sigma$ by the number of element nuclei per unit volume, so $\sigma$ has units of area. The unit usually used to describe nuclear reaction cross sections is the barn; one barn defined as $10^{-24} \mathrm{~cm}^{2}$.

The general equation describing the macroscopic cross-section for a soil containing $n$ different elements is: 


$$
\sum=N_{1} \sigma_{1}+N_{2} \sigma_{2}+\cdots \cdots=\sum_{i=1}^{n} N_{i} \sigma_{i}
$$

where $N_{i}$ is the number of atoms of element $i$ per $\mathrm{cm}^{3}$ of the medium and $\sigma_{i}$ is its microscopic cross section at the specified energy level (and for the specified interaction; the designations, $\sigma_{s}$ and $\sigma_{a}$, are used for scattering and absorption interactions respectively). With Avogadro's number $\left(N_{0}=6.02 \times 10^{23}\right)$, equation (5) can be rewritten more conveniently as

$$
\sum=6.02 \times 10^{-3} \rho \sum_{i=1}^{n} \frac{w_{i} \sigma_{i}}{A_{i}}
$$

where $A_{i}$ is the atomic weight of the $i$ th element (see Table 2), $w_{i}$ is the percentage weight of the $i$ th element, and $\rho$ is the density of the medium in $\mathrm{g} / \mathrm{cm}^{3}$.

In practically every case known, the microscopic cross section $(\sigma)$ depends on the energy level of the neutrons. To illustrate this, the microscopic elastic scattering cross sections for hydrogen and oxygen are shown in Figure 2. For these elements, elastic scattering cross sections are almost constant over the middle energy levels, but decrease at higher neutron energies (and may vary irregularly; see for example, oxygen), while at low energies scattering usually increases.

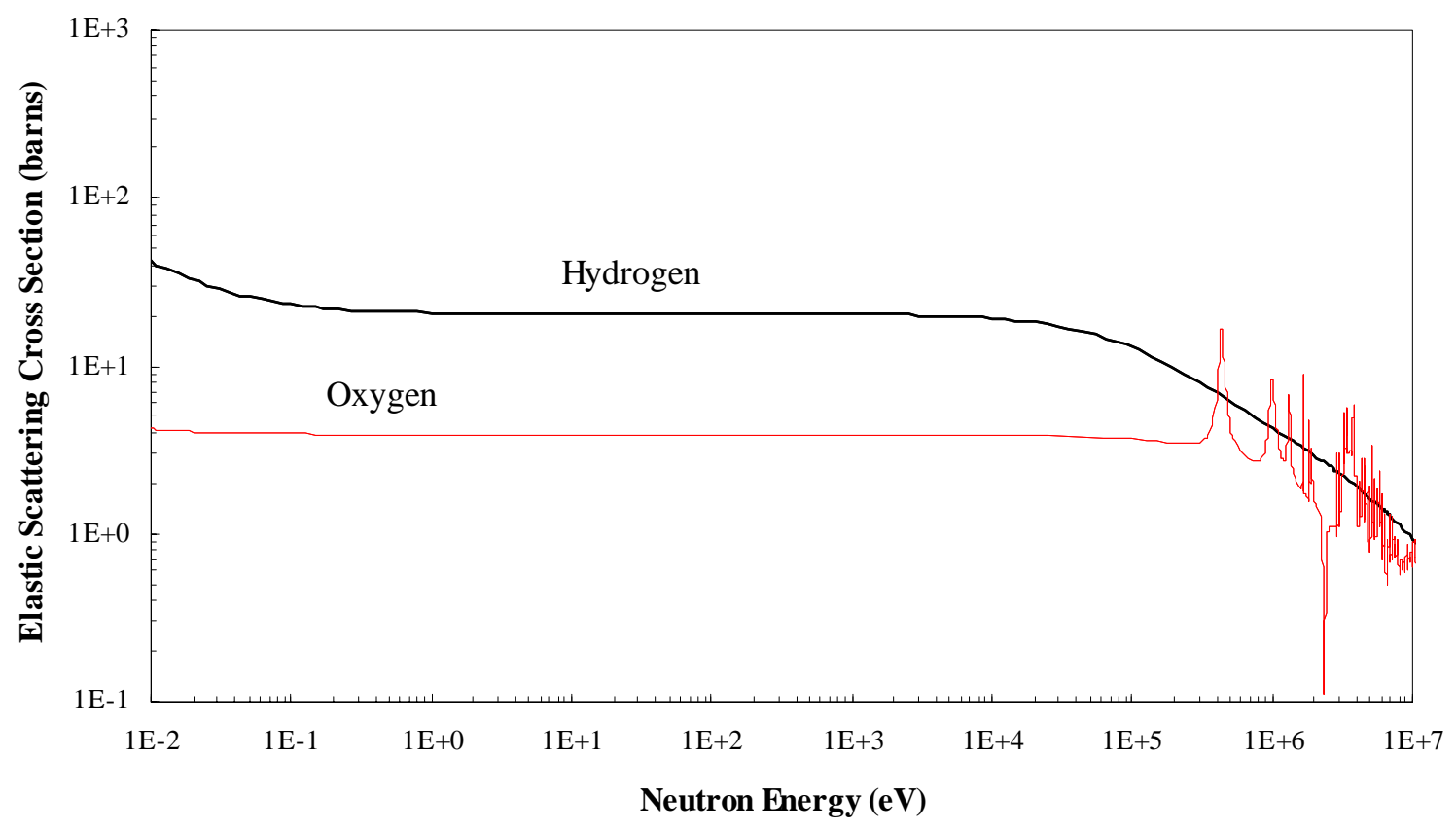

Figure 2. Elastic scattering cross section of ${ }^{1} \mathrm{H}_{1}$ and ${ }^{16} \mathrm{O}_{8}$ as function of neutron energy after Stacey (2001) 
As noted previously, the absorption of fast neutrons can usually be neglected in ordinary soils since absorption rapidly decreases for energies above the thermal range. At low energy levels, absorption is approximately inversely related to the neutron velocity (i.e., $\sigma_{a}$ is proportional to $1 / v)$. This behaviour is often referred to as the ' $1 / v$ law' (Iliffe, 1982).

The theoretical determination of nuclear cross sections for scattering and absorption interactions is a complex problem, and so the cross sections are usually measured experimentally. A list of microscopic thermal absorption and microscopic scattering cross sections of the 20 most common elements in most soils is shown in Table 2 (data for other elements can be found in Stacey (2001)). The 2200 superscript represents the neutron velocity $(v)$ when thermalised (i.e. at 0.025 $\mathrm{eV}, v=2200 \mathrm{~m} / \mathrm{s})$.

Table 2. Microscopic thermal cross sections (from Stacey, 2001)

\begin{tabular}{cccc}
\hline Element & $\begin{array}{c}\text { Atomic mass } \\
A\end{array}$ & $\begin{array}{c}\sigma_{\mathrm{a}}^{2200} \\
\text { barn }\end{array}$ & $\begin{array}{c}\bar{\sigma}_{s} \\
\text { barn }\end{array}$ \\
\hline $\mathrm{H}$ & 1.008 & 0.332 & $20.49^{*}$ \\
$\mathrm{Li}$ & 6.94 & 71 & 1.4 \\
$\mathrm{~B}$ & 10.82 & 755 & 4 \\
$\mathrm{C}$ & 12.011 & 0.00373 & 4.8 \\
$\mathrm{~N}$ & 14.008 & 1.88 & 10 \\
$\mathrm{O}$ & 16 & 0.0002 & 4.2 \\
$\mathrm{Na}$ & 22.99 & 0.505 & 4 \\
$\mathrm{Mg}$ & 24.32 & 0.069 & 3.6 \\
$\mathrm{Al}$ & 26.98 & 0.241 & 1.4 \\
$\mathrm{Si}$ & 28.09 & 0.16 & 1.7 \\
$\mathrm{P}$ & 30.975 & 0.2 & 5 \\
$\mathrm{~S}$ & 32.066 & 0.52 & 1.1 \\
$\mathrm{Cl}$ & 35.457 & 33.8 & 16 \\
$\mathrm{~K}$ & 39.1 & 2.07 & 1.5 \\
$\mathrm{Ca}$ & 40.08 & 0.44 & 3 \\
$\mathrm{Ti}$ & 47.9 & 5.8 & 4 \\
$\mathrm{Mn}$ & 54.94 & 13.2 & 2.3 \\
$\mathrm{Fe}$ & 55.85 & 2.62 & 11 \\
$\mathrm{Co}$ & 58.94 & 38 & 7 \\
$\mathrm{Cd}$ & 112.41 & 2450 & 7 \\
$\mathrm{H}{ }_{2} \mathrm{O}$ & 18.016 & 0.664 & - \\
\hline
\end{tabular}

*From Mughabghab et al (1981).

\subsection{Neutron Flux}

The 'neutron flux' $\phi$ is a very significant quantity in all studies concerning neutrons. For a prescribed energy level it is defined as (Glasstone and Edlund, 1957): 


$$
\phi=n v
$$

where $n$ is the neutron density (i.e. the number of neutrons per cubic centimeter) and $v$ is the neutron velocity (e.g. $\mathrm{cm} / \mathrm{sec}$.). Hence the units of neutron flux are (neutrons) $(\mathrm{cm}) /\left(\mathrm{cm}^{3}\right)(\mathrm{sec})$, that is, neutrons $/ \mathrm{cm}^{2}-\mathrm{sec}$ (as is suggested by the name 'neutron flux'). However, one should not be misled by the usual interpretation that the flux represents the number of neutrons crossing a unit area per unit time. This interpretation is correct only for a collimated beam (all neutrons traveling in one direction) with the unit area taken normal to the travel direction. In a neutron gauge system, the neutrons may travel in all directions. The general interpretation of the flux is that the product, $n v$, represents the sum of the distances traveled by all the neutrons in one second in a unit volume. This interpretation is independent of neutron direction.

Since neutron cross sections are energy-dependent, the neutron flux is often expressed as a function of energy. The total flux over the energy range $E_{1}$ to $E_{2}$ is then,

$$
\phi=\int_{E_{1}}^{E_{2}} n(E) v(E) d E=\int_{E_{1}}^{E_{2}} \phi(E) d E
$$

where $n(E)$ defines the number of neutrons per unit volume per unit energy interval. Hence $n(E) d E$ is the number of neutrons per unit volume in the energy interval from $E$ to $E+d E$.

\subsection{Neutron current}

Another important quantity for describing neutron behaviour is the neutron current, $\boldsymbol{J}$ (also known as neutron current density). Using Fick's law of diffusion, the net current of monoenergetic neutrons (i.e. neutrons of uniform energy) can be written as (Isbin, 1963; Stacey, 2001):

$$
\boldsymbol{J}=-D \operatorname{grad} \phi
$$

where the neutron current vector $\boldsymbol{J}$ is the net number of neutrons flowing in a given direction in unit time through a unit area normal to the direction of flow, and $D$ is the diffusion coefficient.

It should be noted that the units of neutron flux and neutron current are the same (neutrons per square centimeter per second), but the neutron current is a vector quantity, while the neutron flux is a scalar quantity. Equation (9) then implies that the diffusion coefficient has units of length 
(cm). When the energy spectrum of the neutrons is considered, the neutron current becomes a function of the energy, as does the neutron flux $(\phi)$ and neutron density $(n)$.

\subsection{Neutron Diffusion Equation}

When neutrons have slowed to thermal energies, their spatial movement is quite similar to the diffusion of gases, except that their lifetime is limited by absorption (IAEA, 1970). After high energy neutrons are emitted by the source and diffuse outward through the soil, a fraction of the slow neutrons rebound back towards the probe and are absorbed by the nucleus of the gas in the detector, giving rise to a signal that, after processing, is known as the 'neutron count'. The detector only measures slow neutrons.

It can be summarized that in a neutron moisture probe the fast, high energy neutrons emitted from the source undergo simultaneously the processes of transport (diffusion) and slowing-down (themalization). Therefore a model describing neutron moisture probe behaviours must take into account the complete energy spectrum of neutrons, from their initial fast state to their thermalized state. A straightforward and practical approach is to subdivide the continuous energy spectrum of all neutrons into a number of discrete energy groups so that each energy group can, to reasonable approximation, be treated as monoenergetic with constant parameters. A set of simultaneous diffusion equations then covers the whole neutron spectrum from fast down to thermal, a representation known as 'multigroup diffusion theory' (Iliffe, 1982).

For a given neutron energy group, the neutron balance (or conservation) equation under steadystate conditions is expressed as:

$$
\overbrace{(-\nabla \boldsymbol{J})}^{\text {Leakage }}+\overbrace{\left(\sum_{s l_{i}} \phi_{i}+\Sigma_{a_{i}} \phi_{i}\right)}^{\text {Sink }}=\overbrace{\left(S \text { or } \Sigma_{s l_{i-1}} \phi_{i-1}\right)}^{\text {Source }}
$$

From this neutron balance, the neutron diffusion equation for an n-group diffusion model can be written in the form: 


$$
\left.\begin{array}{ll}
D_{1} \nabla^{2} \phi_{1}-\Sigma_{s l_{1}} \phi_{1}-\Sigma_{a_{1}} \phi_{1}+S=0 & E_{1} \geq E>E_{2} \\
D_{2} \nabla^{2} \phi_{2}+\Sigma_{s l_{1}} \phi_{1}-\Sigma_{s l_{2}} \phi_{2}-\Sigma_{a_{2}} \phi_{2}=0 & E_{2} \geq E>E_{3} \\
D_{3} \nabla^{2} \phi_{3}+\Sigma_{s l_{2}} \phi_{2}-\Sigma_{s l_{3}} \phi_{3}-\Sigma_{a_{3}} \phi_{3}=0 & E_{3} \geq E>E_{4} \\
\ldots \ldots & \ldots \ldots \\
D_{n} \nabla^{2} \phi_{n}+\Sigma_{s l_{n-1}} \phi_{n-1}-\Sigma_{a_{n}} \phi_{n}=0 & E_{n} \geq E
\end{array}\right\}
$$

where $S$ is the high energy neutron source term, $D_{i}$ is the diffusion coefficient for the $i$ th energy group, $\Sigma_{\mathrm{si}_{\mathrm{i}}}$ is the slow-down cross-section, $\Sigma_{\mathrm{a}_{\mathrm{i}}}$ is the macroscopic absorption cross-section and $E_{i}$ is the energy interval. The unknown quantities $\phi_{l}$, are the neutron flux fields to be found (defined in Equation (8)). The groups are numbered from 1, for the highest energy, to $n$, for the lowest (i.e. thermal) energy. Clearly the larger the number of energy groups, the greater the accuracy, since one is attempting to represent the actual continuous energy distribution in a material by a finite number of discrete groups. In an index notation, equations (11) are denoted,

$$
\frac{\partial}{\partial x_{i}}\left(D_{l k} \frac{\partial \phi_{k}}{\partial x_{i}}\right)-a_{l k} \phi_{k}=-f_{l}
$$

where the standard summation convention applies. $a_{l k}$ is a matrix of the slow-down and absorption cross sections. It is noted that the diffusion coefficient in equation (12) represents a constant (i.e. for an isotropic material), and is not a second order tensor quantity.

In its crudest approximation, 'multigroup diffusion theory' reduces to a single group. Single group theory assumes that all diffusion and absorption of neutrons occur in a single energy, that is, at the thermal energy. Obviously, this model is not a good model for neutron probe analysis. The twogroup diffusion model breaks the energy spectrum of neutrons into two separate groups (i.e. fast and thermal groups), while for the three-group theory, the fast neutrons are further split into an upper and lower fast groups. Analytical solutions to equation (11) or (12) are available for one, two and three group diffusion theory, assuming a point source situated in an infinite homogeneous medium.

Two group theory was used by Haahr and Olgaard (1965) to determine a so-called 'sphere of importance'. Olgaard (1965) also used three-group diffusion theory as an improvement on the two-group theory calculations and achieved reasonable agreement with some experimental measurements in various soil types. Based on the three-group model developed by Olgaard (1965), Elder and Rasmussen (1994) obtained a calibration equation between neutron counts and 
water content in an unsaturated tuff. The three group approximation was also applied by Morris and Williams (1990) to develop a moisture content calibration for coal mine tailings.

While these solutions have served to assist in neutron probe calibration, the assumptions made in deriving the analytic solutions clearly ignore the access tube geometry, probe and detector geometry, the spatially variable soil composition, and the boundary conditions encountered in practice.

In this study, a numerical model based on seven-group diffusion theory has been developed to give a better physical description of the problem and to improve upon previous results. Seven group diffusion theory (whose upper and lower energy limits are given in Table 3), was found to be sufficiently accurate to describe neutron slowing down and diffusion in a neutron gauge. As far as the authors are aware, this is the first time a seven group diffusion theory has been applied to a neutron probe analysis.

Table 3. Upper and lower energy limits, $E_{i}$, used in the numerical model

\begin{tabular}{ccc}
\hline Group & Upper limit & Lower limit \\
\hline 1 & $4.5 \mathrm{MeV}$ & $4.0 \mathrm{MeV}$ \\
2 & $4.0 \mathrm{MeV}$ & $3.0 \mathrm{MeV}$ \\
3 & $3.0 \mathrm{MeV}$ & $2.0 \mathrm{MeV}$ \\
4 & $2.0 \mathrm{MeV}$ & $1.0 \mathrm{MeV}$ \\
5 & $1.0 \mathrm{MeV}$ & $0.1 \mathrm{MeV}$ \\
6 & $0.1 \mathrm{MeV}$ & $1.44 \mathrm{eV}$ \\
7 & $1.44 \mathrm{eV}$ & $5 \mathrm{k} T_{n}^{*} \mathrm{eV}$ \\
\hline
\end{tabular}

$* T_{n}$ is the neutron temperature.

\subsection{Calculation of Diffusion Coefficient, Slow-down and Absorption Cross Sections}

To solve the neutron diffusion equation (11), the diffusion coefficients $D_{i}$, the slow-down cross sections $\Sigma_{\mathrm{sl}_{\mathrm{i}}}$, and the absorption cross-section $\Sigma_{\mathrm{a}_{\mathrm{i}}}$ have to be first estimated. As mentioned previously, absorption rapidly decreases for energies above the thermal energy. Therefore absorption of fast neutrons can be neglected in moisture gauge studies with little loss in accuracy.

By assuming that the thermal neutrons are distributed according to the Maxwell-Boltzmann distribution law (because the thermal neutrons behave like an ideal gas) and that all absorbers follow the ' $1 / v$ law', then the absorption cross-section for the thermal neutrons is found to be (Olgaard, 1965): 


$$
\sum_{\mathrm{a}}=\frac{\sqrt{\pi}}{2} \sum_{\mathrm{a}}^{2200} \sqrt{\frac{293}{T_{n}}}
$$

where $\sum_{\mathrm{a}}^{2200}$ and $T_{n}$ are the total macroscopic absorption cross section of the medium corresponding to a neutron velocity of $2200 \mathrm{~m} / \mathrm{sec}$ and the neutron temperature respectively. $T_{n}$ is obtained from the following relation (Weinberg and Wigner, 1958):

$$
T_{n}=T_{m}\left(1+0.92 \frac{\sum_{\mathrm{a}}^{2200}}{\sum_{\mathrm{s}} / A} \sqrt{\frac{293}{T_{m}}}\right)
$$

It can be seen that the neutron temperature is always higher than the physical temperature, $T_{m}$ (in ${ }^{o} \mathrm{~K}$ ), of the medium in which neutrons diffuse, and this difference is dependent on the macroscopic absorption cross section of the medium. $\sum_{\mathrm{a}}^{2200}$ may be calculated based on the experimentally estimated microscopic cross sections given in Table 2, viz,

$$
\sum_{\mathrm{a}}^{2200}=n_{\mathrm{H}_{2} \mathrm{O}} \sigma_{a, H_{2} \mathrm{O}}^{2200}+\sum_{1}^{n} n_{i} \sigma_{a, i}^{2200}
$$

where $n_{i}$ and $n_{\mathrm{H}_{2} \mathrm{O}}$ are the number of atoms of a specific chemical element and the number of water molecules per cubic centimeter respectively. $n_{i}$ is defined as,

$$
n_{i}=\frac{6.02 \times 10^{23}}{A_{i}} \times \rho_{s} \times \frac{w_{i}}{100}
$$

where $\rho_{s}$ is the dry density of the soil and $A_{i}$ is the atomic mass given in Table 2. The mass percentage of the $i$ th element, $w_{i}$, is usually determined from the chemical analysis of the soil material. If the volume fraction of water in the soil (in per cent of the total volume) is equal to $V_{w}$, $n_{\mathrm{H}_{2} \mathrm{O}}$ is given by (Olgaard, 1965),

$$
n_{\mathrm{H}_{2} \mathrm{O}}=\frac{6.02 \times 10^{23}}{18.016} \times \rho_{w} \times \frac{V_{w}}{100}
$$


The density of water, $\rho_{w}$, shown in equation (17) is a function of water temperature, $t_{m}$, in degrees centigrade. This relationship is taken to be,

$$
\rho_{w}=0.9998+6.296 \times 10^{-5} t_{m}-8.201 \times 10^{-6} t_{m}^{2}+4.826 \times 10^{-3} t_{m}^{3}
$$

For the calculation of the thermal neutron temperature (equation (14)), a value of $\sum_{\mathrm{s}} / A$ may be estimated using the following formula,

$$
\sum_{s} / A=n_{H_{2} O}\left(2 \bar{\sigma}_{s, H} / A_{H}+\bar{\sigma}_{s, O} / A_{O}\right)+\sum_{1}^{n} n_{i} \bar{\sigma}_{s, i} / A_{i}
$$

For thermalized neutrons, both the macroscopic scattering and absorption cross sections are important. The macroscopic thermal scattering cross sections of the soil elements are calculated with the assumption that the microscopic scattering cross sections of all elements in the material are independent of neutron energy; but the contribution to $\sum_{\mathrm{s}}(1-\bar{\mu})$ from water is assumed to be dependent on the neutron temperature (see equation 20). This is expressed by:

$$
\sum_{s}(1-\bar{\mu})=2.156 \times\left(0.047+0.953 \sqrt{\frac{293}{T_{n}}}\right) \times \rho_{w} \frac{v_{w}}{100}+\sum_{1}^{n} n_{i} \bar{\sigma}_{s, i}\left(1-\frac{2}{3 A_{i}}\right)
$$

Once the macroscopic absorption and scattering cross sections have been determined, the macroscopic transport cross section of the medium, $\Sigma_{\text {tr }}$, and the thermal diffusion coefficient, $D_{t h}$, are calculated using (IAEA, 1970)

$$
\begin{aligned}
& \sum_{t r}=\sum_{s}(1-\bar{\mu})+\sum_{a} \\
& D_{t h}=\frac{1}{3 \sum_{t r}}
\end{aligned}
$$

For fast neutrons, the scattering interaction is of primary importance (i.e. absorption is small and so neglected). The slow-down cross sections are determined using (Iliffe, 1982),

$$
\Sigma_{s l_{i}}=\frac{\left(\xi \Sigma_{s}\right)_{i}}{\ln \left(E_{i} / E_{i+1}\right)}
$$


The product, $\xi \Sigma_{\mathrm{s}}$, is called the 'macroscopic slowing down power' and given by

$$
\left(\xi \sum_{s}\right)_{i}=n_{H_{2} O}\left[2\left(\bar{\xi} \sigma_{s}\right)_{H, i}+\left(\bar{\xi} \sigma_{s}\right)_{O, i}\right]+\sum_{j=1}^{n} n_{i}\left(\bar{\xi} \sigma_{s}\right)_{j, i}
$$

where $\Sigma_{\mathrm{s}_{\mathrm{i}}}$ is the macroscopic cross-section for scattering. Considering the forward biases from the 'center of mass correction' and the anisotropic scattering, the energy dependent expressions for $\bar{\xi}$ and $\bar{\mu}$ are taken to be (Weinberg and Wigner, 1958):

for $0.07 A_{i}^{2 / 3} \leq 0.2$

$$
\begin{aligned}
& \bar{\mu}=\frac{2}{3 A}+0.07 A^{2 / 3} E\left(1-\frac{3}{5 A^{2}}\right) \\
& \bar{\xi}=\xi-0.21 A^{2 / 3} E \times\left(\frac{\xi}{a}-0.5\right)
\end{aligned}
$$

for $0.07 A^{2 / 3}>0.2$

$$
\begin{aligned}
& \bar{\mu}=\frac{2}{3 A}+0.2\left(1-\frac{3}{5 A^{2}}\right) \\
& \bar{\xi}=\xi-0.6 \times\left(\frac{\xi}{a}-0.5\right)
\end{aligned}
$$

Fast group constants, $\Sigma_{\mathrm{tr}}$ and $D_{i}$, are now obtained from

$$
\begin{gathered}
\sum_{t r}=\left[\sum_{s}(1-\bar{\mu})\right]_{i}=n_{H_{2} O}\left[2\left(\sigma_{s}(1-\bar{\mu})\right)_{H, i}+\left(\sigma_{s}(1-\bar{\mu})\right)_{O, i}\right]+\sum_{j=1}^{n} n_{i}\left[\sigma_{s}(1-\bar{\mu})\right]_{j, i} \\
D_{i}=\frac{1}{3 \sum_{t r}}
\end{gathered}
$$

The fast microscopic scattering cross sections $\left(\sigma_{s}\right)$ for each energy group, can be estimated using the figures in Hughes and Schwartz (1958) or Mughabghab et al. (1981). As noted previously and from the cross section curves given in Hughes and Schwartz (1958), it can be seen that the cross 
sections for fast neutrons may vary in a highly irregular way with energy level, having many high sharp peaks. If required, more accurate cross section data can be obtained from the MIRANDA code developed by the Australian Nuclear Science and Technology Organisation (Robinson and Harrington, 1998), which contains the cross section library for all elements over the 200 energy groups (covering the range $15.5 \mathrm{MeV}$ to $1 \times 10^{-5} \mathrm{eV}$ ) based on the US data file ENDF/B-VI.

\subsection{Material Interface and Boundary Conditions}

In order to solve the general governing equations (11) or (12), the following material interface and boundary conditions need to be specified.

\section{(a) Interface between two different mediums}

At an interface between two media with different diffusion properties (for example, between the detector and soil or between two different soils), both neutron flux and neutron net current must be continuous across the interface. If two media are denoted by the symbols $A$ and $B$, then the required boundary conditions for neutrons with a particular energy range are simply,

$$
\begin{gathered}
\phi_{A}=\phi_{B} \\
J_{A}=J_{B}
\end{gathered}
$$

(b) Soil-atmosphere boundary

At the top surface of a soil profile, there is a flow of neutrons out of the surface but there is practically no flow from the atmosphere back into the surface. The correct boundary condition is clearly that there are no neutrons returned from the atmosphere to the soil (Figure 3). Generally, the net neutron current, $\boldsymbol{J}$, represents the balance between the currents in the positive (outward) and negative (inward) directions; according to neutron diffusion theory these are defined as:

$$
\begin{aligned}
& \boldsymbol{J}_{\text {out }}^{+}=\frac{\phi_{i}}{4}-\frac{1}{2} \vec{n} \cdot\left(D_{i} \nabla \phi_{i}\right) \\
& \boldsymbol{J}_{\text {in }}^{-}=\frac{\phi_{i}}{4}+\frac{1}{2} \vec{n} \cdot\left(D_{i} \nabla \phi_{i}\right)
\end{aligned}
$$


where $\phi_{i}$ and $D_{i}$ are the neutron flux and the diffusion coefficient for the $i$ th energy group respectively. The difference between these two components gives the net neutron current, in agreement with equation (9). Since there is no scattering back of neutrons from the air into the soil medium, the negative component of the neutron current for all seven energy groups is zero and so:

$$
\boldsymbol{J}_{\text {in }}^{-}=\frac{\phi_{i}}{4}+\frac{1}{2} \vec{n} \cdot\left(D_{i} \nabla \phi_{i}\right)=0
$$

Since the flux $\phi_{i}$ at the boundary is positive, the flux gradient $\left(\nabla \phi_{1}\right)$ must be negative, as indicated schematically in Figure 3. Therefore if the neutron flux is extrapolated into the air, as shown in Figure 3, it will vanish at some distance $e$ beyond the boundary.

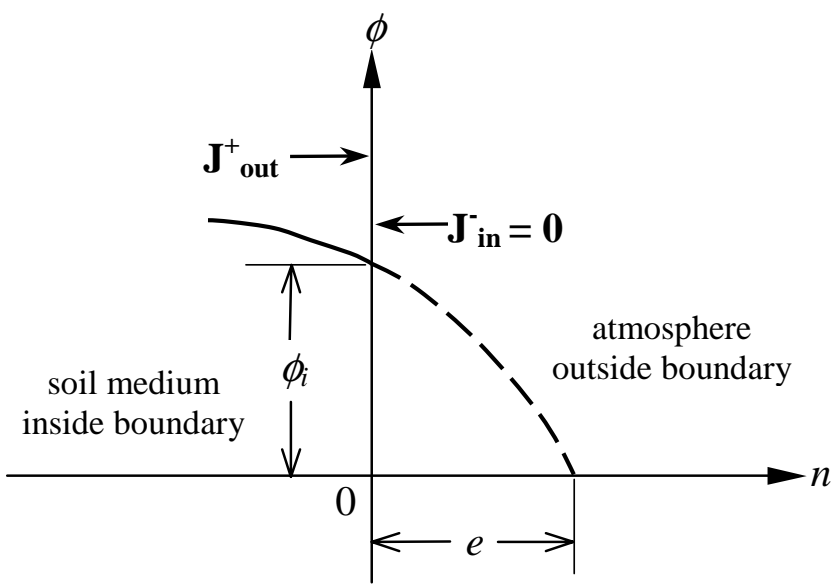

Figure 3. Interface between the soil and atmosphere

It can be seen that modeling the response of a neutron gauge is a complex problem. Analytical solutions are possible only for the very simplest cases, and consequently numerical methods need to be adopted. Given that the problem geometry may be irregular, that there may be multiple materials with varying properties, and a range of different types of boundary conditions, it is apparent that solving the neutron diffusion equations is ideally suited to the finite element method.

\section{NUMERICAL MODEL}

\subsection{The Finite Element Formulation}

The governing differential equation for multi-group neutron diffusion with boundary conditions can be written as 


$$
\begin{cases}-\frac{\partial}{\partial x_{i}}\left(D_{l k} \frac{\partial \phi_{k}}{\partial x_{i}}\right)+a_{l k} \phi_{k}=f_{l} & \text { in } \Omega \\ n_{i}\left(D_{l k} \frac{\partial \phi_{k}}{\partial x_{i}}\right)+q_{l k} \phi_{k}=g_{l} & \text { on } \partial \Omega \\ h_{l k} \phi_{k}=r_{l} & \text { on } \partial \Omega\end{cases}
$$

where $\Omega$ is the domain and $\partial \Omega$ is the boundary of the domain. The second equation is referred to as a generalized Neumann boundary condition, and the third equation as a Dirichlet boundary condition. For two-dimensional or axi-symmetric problems the space index $i$ ranges from 1 to 2 . The component indices $k$ and $l$ range from 1 to $N$; where $N$ is the number of coupled second-order partial differential equations; and $n_{i}$ is the $i$ th component of the outward normal vector.

Equation (36) is solved using the finite element spatial discretization procedure. Without restricting the generality, we assume generalised Neumann conditions on the whole boundary. Multiplying an arbitrary test function $v$ by equation (36) and applying Gauss's theorem, we find

$$
\int_{\Omega}\left(\left(D_{l k} \frac{\partial \phi_{k}}{\partial x_{i}}\right) \frac{\partial v}{\partial x_{i}}+a_{l k} \phi_{k} v\right) d x+\int_{\partial \Omega} q_{l k} \phi_{k} v d s=\int_{\Omega} f_{l} v d x+\int_{\partial \Omega} g_{l} v d s
$$

This equation defines the weak or variational form of the governing differential equation. Obviously, any solution of the differential equation is also a solution of the variational problem. In order to obtain the weak solution of equation (37), we need to project the weak form of the differential equation onto a finite dimensional function space (i.e., a set of continuous, piecewise linear functions on a triangulation $\tau$ of the domain $\Omega$ ). Introducing the space discretization

$$
\phi_{k}(x) \approx \sum_{I=1}^{N_{p}} \psi_{I, k} N_{I}(x)
$$

where $N_{I}$ are the shape functions, $\psi_{I, k}$ are the nodal values of the unknown, and $N_{p}$ is the number of nodes in an element. By setting the test functions $v$ equal to the shape function $N_{J}$ in equation (37), we obtain a system of equations in matrix form as:

$$
(\boldsymbol{C}+\boldsymbol{A}+\boldsymbol{Q}) \psi=\boldsymbol{F}+\boldsymbol{G}
$$


where

$$
\begin{aligned}
& C_{(J, l),(I, k)}=\Sigma \int_{\tau} D_{l k} \frac{\partial N_{I}}{\partial x_{i}} \frac{\partial N_{J}}{\partial x_{i}} d x \\
& A_{(J, l),(I, k)}=\Sigma \int_{\tau} a_{l k} N_{I} N_{J} d x \\
& Q_{(J, l),(I, k)}=\Sigma \int_{\partial \tau} q_{l k} N_{I} N_{J} d s \\
& F_{(J, l)}=\Sigma \int_{\tau} f_{l} N_{J} d x \\
& G_{(J, l)}=\Sigma \int_{\partial \tau} g_{l} N_{J} d s
\end{aligned}
$$

The sign $\Sigma$ indicates the summation over all elements, and $\psi$ is an $N_{p} N$-vector containing the numbers $\psi_{I, k}$ :

$$
\boldsymbol{\psi}=\left[\psi_{1,1} \psi_{2,1} \cdots \psi_{N_{p}, 1} \psi_{1,2} \psi_{2,2} \cdots \psi_{N_{p}, 2} \cdots \psi_{1, N} \cdots \psi_{N, N}\right]^{T}
$$

Solution of equation (39) results in a set of nodal flux values for each neutron energy group in the domain of interest.

\subsection{The Two Dimensional Model}

The source, detector and surrounding soils were modelled in an axisymmetric geometry as shown in Figure 4. This geometry is a reasonable representation of the physical arrangement of modern neutron moisture gauges. The volume of the soil shown in Figure 4 exceeds the radius of

influence of the neutron probe since the effective volume 'sensed' by a neutron probe is approximately a sphere of radius 20 to $70 \mathrm{~cm}$, increasing with decreasing moisture content (because at low water contents, the fast neutrons have to travel greater distances to undergo scattering interactions and so become thermalized). 
symmetric axis

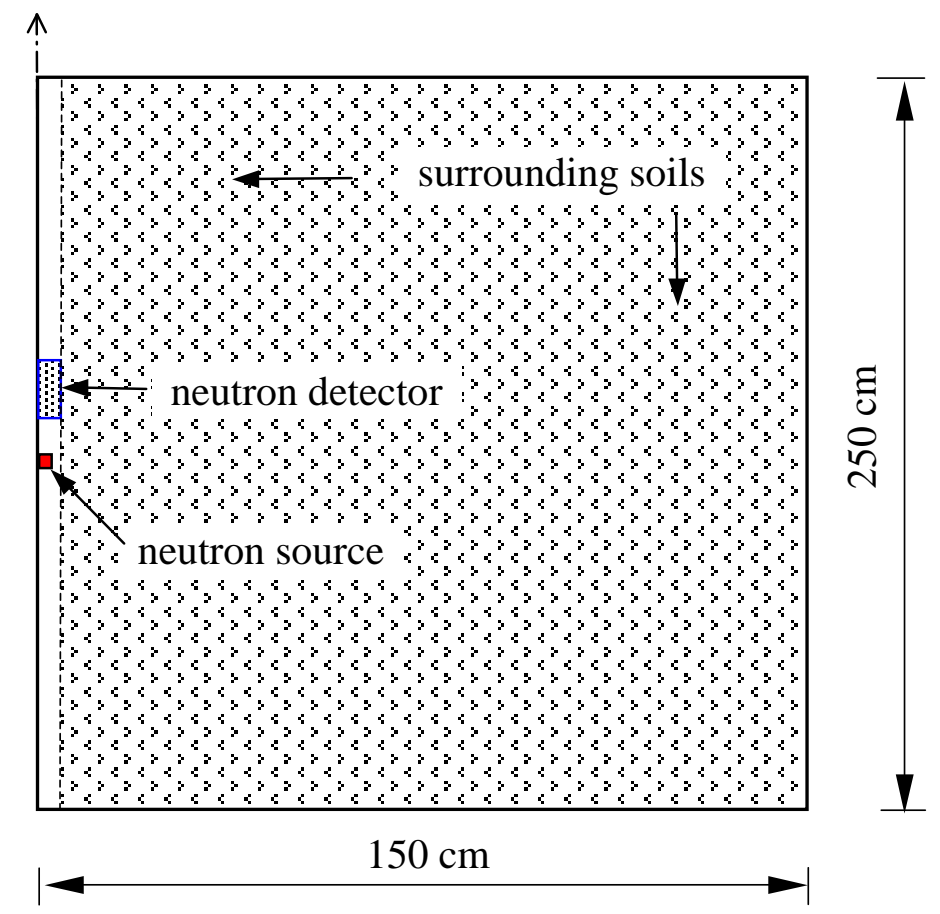

Figure 4. The cylindrical system used for numerical analysis (not to scale).

The two-dimensional axisymmetric finite element analysis was carried out to calculate the thermal flux distribution in the system. A total of 4073 three-node triangular elements were used in the analysis.

In order to include the effect of the detector in the analysis, the detector was treated as a separate region. The detector is usually a gas with a very large absorption cross-section. The most widely used detector gasses are ${ }^{3} \mathrm{He}$ and ${ }^{10} \mathrm{BF}_{3}$. Their thermal absorption cross-sections may be estimated from the following equation,

$$
\sum_{a, D}=\frac{0.6023 \times 10^{24}}{22.41 \times 10^{3}} \frac{p}{760} \frac{273}{T_{D}} \frac{E}{100} \frac{\sqrt{\pi}}{2} \sigma_{a}^{2200} \times 10^{-24} \sqrt{\frac{293}{T_{n}}}\left(\mathrm{~cm}^{-1}\right)
$$

where $p$ is the pressure of the gas in the detector (in $\mathrm{mm} \mathrm{Hg}$ ), $T_{D}$ is the detector temperature (in degrees Kelvin), $E$ is the percentage of the detector gas in the detector, and $T_{n}$ is the neutron temperature. For the ${ }^{10} \mathrm{BF}_{3}$-filled and ${ }^{3} \mathrm{He}$-filled proportional counters, $\sigma_{\mathrm{a}}^{2200}$ may be taken as 3813 barns and 5330 barns respectively.

It should be noted that the effects of the access tube and absorption in the neutron source are not taken into account in this study. Although the numerical approach described here could include 
these effects, it is believed that their influences on neutron flux at the detector are insignificant (at least for the aluminum access tubes considered here).

Once the distribution of the thermal neutron flux, $\phi_{\mathrm{th}}$, in the neutron detector (as shown in Figure 4) is known, the gauge response (i.e. the number of counts or neutrons detected in a given time) can be obtained by integrating $\phi_{\mathrm{th}} \sum_{\mathrm{a}, \mathrm{D}}$ over the volume of the detector. That is, by evaluating

$$
C R=\int_{v} \phi_{t h} \cdot \sum_{a, D} \cdot T \cdot d v
$$

where $C R$ is the count rate and $T$ is the count period in sec.

\section{VERIFICATION OF THE NUMERICAL MODEL USING LABORATORY EXPERIMENTAL RESULTS}

In order to verify the validity of seven group neutron diffusion model, the numerical results were first checked against laboratory experimental results available in the literature.

The experimental data used for verification purposes were extracted from Olgaard (1965). Neutron probe experiments were conducted on different types of Danish soil by the Agricultural Department at the Riso Establishment. Measurements were performed in a steel drum that was 80 $\mathrm{cm}$ in diameter and $100 \mathrm{~cm}$ in height. For each soil type, measurements were carried out at different water contents. Using the soil elemental composition given in Olgaard (1965), the count rates as function of water volume per cent for Borris soil were predicted by the numerical model. It is noted that Borris soil is described as a 'loamy sand' type of soil. The actual source-detector geometry of the Danish study (i.e. a source situated at the mid-line and just outside the detector) was used in the analysis. The source strength was 79,000 neutrons per second. The results are plotted against the experimental data in Figure 5. As can be seen from Figure 5, when the radius of the cylindrical system in the numerical model (see Figure 4) was reduced from $150 \mathrm{~cm}$ to 40 $\mathrm{cm}$, the predicted count rates agreed very well with the measured values. It is interesting to note that for water contents less than approximately $8 \%$, the calculated count rates are larger than the experimental values. The reason for the discrepancy is that the size of the drum container used in the experiments was too small to prevent neutron leakage out of the container. Similar conclusions about neutron leakage in small-diameter containers at low water contents have been also reported by other researchers (Olgaard, 1965; Olgaard and Haahr, 1967; Elder et al. 1994). 


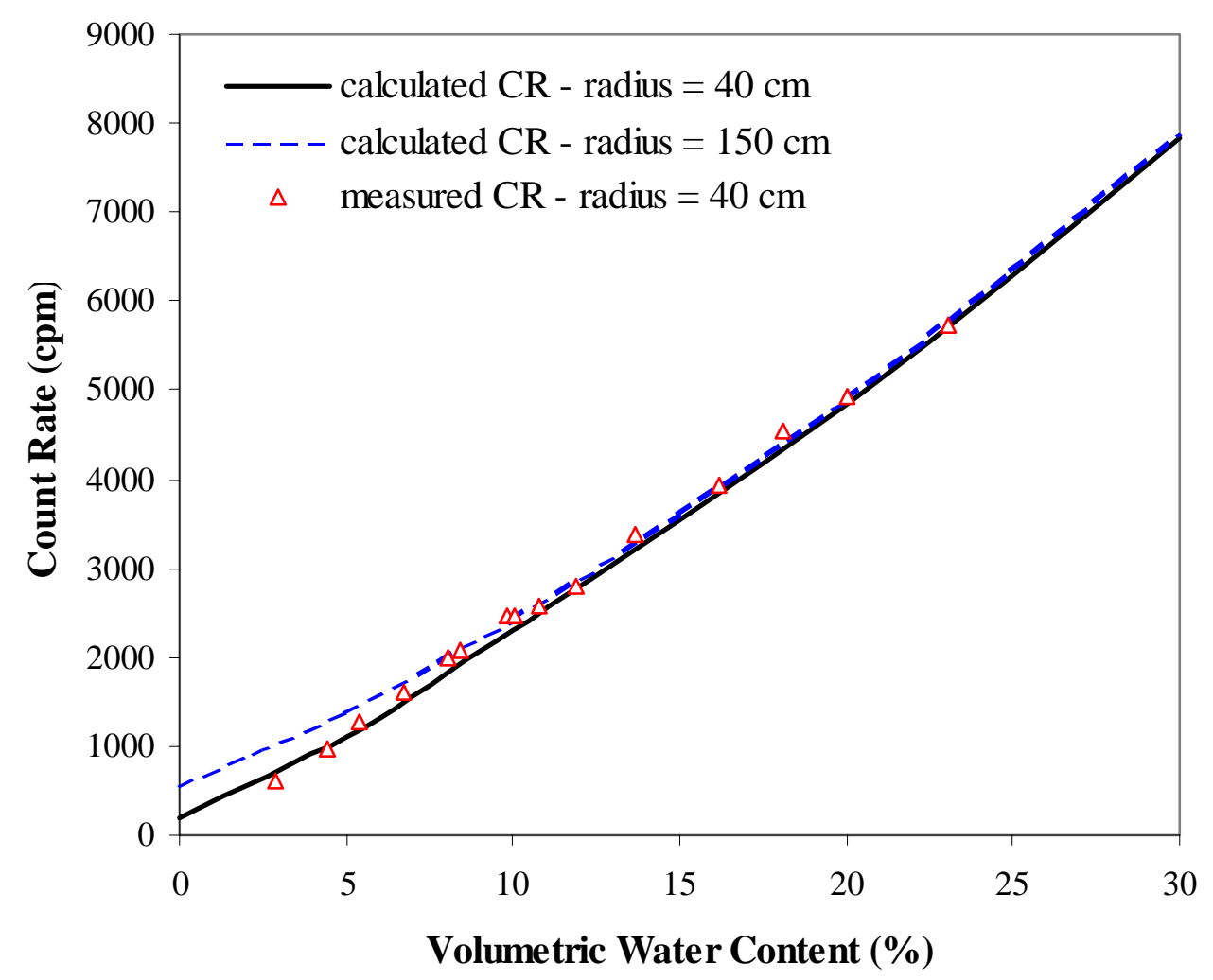

Figure 5. Count rate as a function of water volume per cent for Borris soil - comparison of the results predicted by the numerical model with experimental results.

\section{VERIFICATION OF THE NUMERICAL MODEL USING FIELD EXPERIMENTAL RESULTS}

\subsection{Soil and site description}

The site selected for a field study is located on grassed rangeland some $10 \mathrm{~km}$ west of the city of Newcastle, Australia. The soil profile across the site is relatively uniform and can be generally described as $250 \mathrm{~mm}$ silty clay topsoil underlain by high plasticity clay to a depth of approximately $1.2 \mathrm{~m}$, then a medium plastic silty clay to approximately $1.8 \mathrm{~m}$, where highly weathered siltstone is encountered. The Maryland site classification for reactivity following the Australian Standard for Residential Slab and Footings (AS2870, 1996) is H (i.e. highly reactive, with a predicted open uncovered ground movement ranging between 40 to $70 \mathrm{~mm}$ ). The instrumentation installed at the site includes: automatic weather station, surface movement probes, sub-surface movement probes, neutron probe, gypsum blocks, in situ filter paper devices, thermocouples, and piezometers. Detailed description of the testing site can be found in Fityus et al. (2001). 


\subsection{Chemical composition of the Maryland soils}

In order to carry out the numerical analysis, it is necessary to first know the elemental composition of the soil with depth. A total of fourteen chemical analyses of the soils at different depths over a $3 \mathrm{~m}$ interval were carried out. Before the analysis, the soil samples were dried at $105{ }^{\circ} \mathrm{C}$. Typical results of the chemical analyses for two depths are listed in Table 4. The list shows the mass percent of each element. It should be noted that the oxygen content in Table 4 was estimated by subtracting the sum of the percentages of all other elements from 100 .

As can be seen from Table 4, about half the elemental weight is oxygen (which occurs as metal and silicon oxides). Oxygen is thus the main contributor to the scattering cross section (other major contributors are $\mathrm{Si}, \mathrm{Al}$ and $\mathrm{Fe}$ ).

The macroscopic scattering and absorption cross-sections are calculated from the elemental composition of the soil. The calculation involves a summation of microscopic cross-sections over all elements in the soil.

Table 4. Composition of dried Maryland soils by mass percent

\begin{tabular}{lll}
\hline Element & Depth $=0.45 \mathrm{~m}$ & Depth $=1.25 \mathrm{~m}$ \\
\hline $\mathrm{H}$ & 0.30 & 0.38 \\
$\mathrm{Li}$ & 0.00166 & 0.00167 \\
$\mathrm{~B}$ & 0.006 & 0.0048 \\
$\mathrm{C}$ & 0.64 & 0.41 \\
$\mathrm{~N}$ & 0.09 & 0.12 \\
$\mathrm{O}$ & 54.17 & 52.51 \\
$\mathrm{Na}$ & 0.32 & 0.56 \\
$\mathrm{Mg}$ & 0.50 & 0.55 \\
$\mathrm{Al}$ & 8.26 & 8.64 \\
$\mathrm{Si}$ & 30.87 & 30.2 \\
$\mathrm{P}$ & 0.013 & 0.013 \\
$\mathrm{~S}$ & 0.09 & 0.09 \\
$\mathrm{Cl}$ & 0.005 & 0.046 \\
$\mathrm{~K}$ & 1.21 & 2.06 \\
$\mathrm{Ca}$ & 0.04 & 0.03 \\
$\mathrm{Ti}$ & 0.37 & 0.37 \\
$\mathrm{Mn}$ & 0.005 & 0.005 \\
$\mathrm{Fe}$ & 3.1 & 4.1 \\
$\mathrm{Co}$ & $\sim 0$ & $\sim 0$ \\
$\mathrm{Cd}$ & $\sim 0$ & $\sim 0$ \\
\hline
\end{tabular}




\subsection{Neutron instruments}

The neutron probe used in this study is a Campbell Pacific Nuclear Model 503 Hydroprobe. Fast neutrons are generated by the probe using a $50 \mathrm{mCi}(1.85 \mathrm{GBq})$ Americium-241-Beryllium source, with a strength of 111,000 neutrons per second. A helium-3 filled proportional counter detector of $13.2 \mathrm{~cm}$ in length and $2.54 \mathrm{~cm}$ in diameter is used to detect thermalized neutrons. The neutron source is situated $3 \mathrm{~cm}$ below the detector. The commercial neutron probe was not modified in any way for the research work described herein.

\subsection{Access Tubes}

Aluminum access tubes of $53 \mathrm{~mm}$ external diameter and $1.9 \mathrm{~mm}$ wall thickness were used in this study. Because of the low thermal cross section of aluminum $\left(\sigma_{\mathrm{a}}=0.23 \mathrm{~b}\right.$ and $\sigma_{\mathrm{s}}=1.4 \mathrm{~b}$, see Table 2), it was considered that aluminum casings would have little effect on the neutron flux. All access tubes are sealed at the bottom. When not in use, the top end of the tube is covered with a cap to prevent the ingress of rain and debris.

\subsection{Field measurement}

In order to calibrate the neutron probe readings with volumetric moisture contents, the soil moisture contents need to be measured independently. To do this, thin-walled steel tubes, $120 \mathrm{~mm}$ in length and $48 \mathrm{~mm}$ in diameter, were used to obtain soil samples from the experimental site. Each tube had a sharpened outward-facing edge to allow easy penetration of the soil. To minimize disturbance, the thin-walled steel tubes were pushed into the soil at a constant penetration rate. After being removed from the ground, the ends of the thin-walled sample tube were wax sealed, and then immediately sealed in an air tight jar to maintain the soil moisture content. The soil volumetric and gravimetric moisture contents and the soil density were determined on each soil sample following standard geotechnical practices.

After the last sample was obtained, an aluminum access tube was installed in the center of the core. The neutron probe was then lowered in the tube and count rates were measured at the same depths where the soil samples had been recovered.

The neutron counts of 16-second duration were taken in this study. The counting time interval of 16-seconds was chosen because it gave sufficiently precise readings in acceptable period of time after comparison with 32, 64 and 256- second counts. 


\subsection{Comparison of numerical and field experimental results}

The results predicted by the numerical model were compared with the field observations at Maryland site. The test site, approximately $80 \mathrm{~m}$ by $25 \mathrm{~m}$, was divided into five distinct zones for study: an open area, a naturally grassed, tree affected area, a $10 \mathrm{~m}$ x $10 \mathrm{~m}$ area with an unloaded flexible cover, and a $10 \mathrm{~m}$ x $10 \mathrm{~m}$ area covered by a loaded reinforced concrete slab. Since 1994, the soil moisture content has been monitored at these zones by the neutron probe method.

The numerical model was used to predict the neutron counts with depth for the open area, based on the system geometry defined in Figure 4 and the chemical analysis of the soils at different depths. The soil bulk density and volumetric moisture contents were determined from the field measurement by the thin-walled steel tube method. The calculated count rate variation with depth is plotted in Figure 6. It can be seen that very good agreement is obtained between the numerical analyses and the field measurements

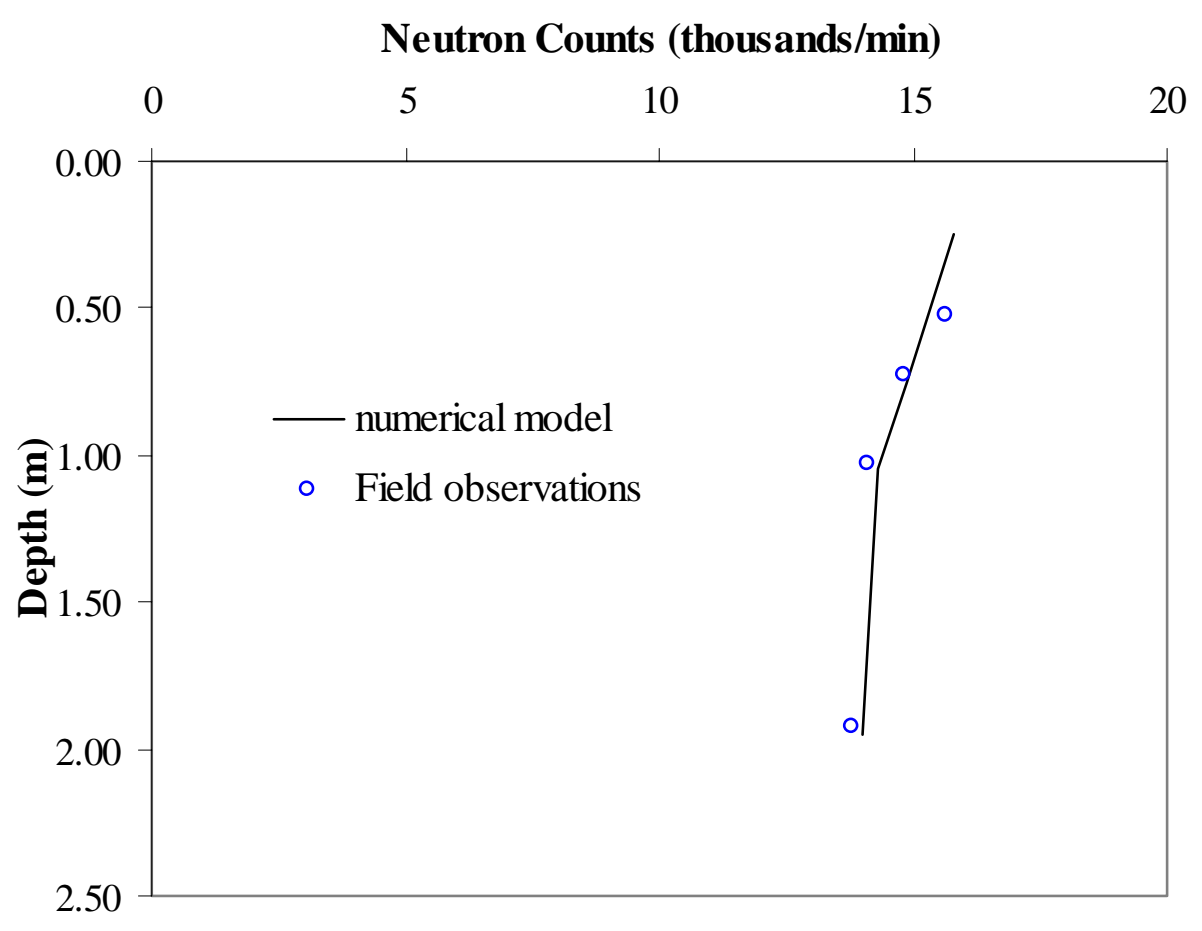

Figure 6. Count rate variation with depth - comparison of the results predicted by the numerical model and the Maryland field measurements. 


\section{CONCLUSIONS}

In this study, a numerical model based on multigroup diffusion theory has been developed to predict the neutron flux distribution in a neutron probe system. Neutron counts predicted by the neutron diffusion model are found to agree favorably with the measured data at a field site. The numerical results also compare very well with the experimental results available in the literature. It is believed that a calibration between neutron counts and moisture content for soils (including unsaturated expansive soil) can be accurately estimated using the proposed numerical model with a minimum of experimental effort. The establishment of a calibration relationship requires that the chemical composition of the soil be determined, and the geometry of the source-detector system be known. The numerical analysis also indicates that large containers are necessary for laboratory calibration to prevent neutrons leaking into the air surrounding the container, and so may be of assistance for laboratory calibration procedures.

An apparent disadvantage of the numerical model is the requirement of a complete elemental analysis of the soil. However, this analysis is now comparatively straightforward using modern analytical chemical methods (e.g. using high resolution inductively coupled plasma mass spectrometry, HR-ICPMS), and is much easier than performing an experimental calibration on soils.

This research has shown that the proposed numerical model is capable of describing the response of a neutron moisture gauge to a degree of sophistication that has not been achieved previously. It is believed that the utilisation of a model such as the one described here can lead to a much better understanding of the distribution of neutron flux in a neutron probe system for any geometry and soil type under a variety of environmental conditions. This greater capacity for interpretation of probe data may open up new applications for the neutron probe. For example, it may be possible to use the neutron probe to monitor rising salt (i.e. sodium chloride) in a groundwater system.

\section{ACKNOWLEDGMENT}

This research has been carried out with financial support from the Mine Subsidence Board of New South Wales. 


\section{REFERENCES}

AS 2870, Residential slab and footings-construction, Standards Association of Australia, Sydney: 1996.

Bell, J. P. and McCulloch, J.S.C., Soil moisture estimation by the neutron method in Britain, Journal of Hydrology, 7, 415-433, 1969.

Carneiro, C. and Jong, E. DE, In situ determination of the slope of the calibration curve of a neutron probe using a volumetric technique, Soil Science, 139 (3), 250-254, 1985.

Chanasyk, D.S. and Naeth, M.A., Field measurement of soil moisture using neutron probes, Can. J. Soil Sci., 76, 317-323, 1996.

Dickey, G.L., Factors affecting neutron gauge calibration, Irrigation and Drainage, Proc. Nat. Conf. Am. Soc. Civil Engineers, Irrigation and Drainage Division, ASCE, 9-20, 1990.

Elder, A.N. and Rasmussen, T.C., Neutron probe calibration in unsaturated Tuff, Soil Sci. Soc. Am. J., 58, 1301-13071, 1994.

Fityus, S.G., Smith, D.W. and Allman, M.A., An expansive soil test site near Newcastle, Accepted by ASCE Journal of Geotechnical and Geoenvironmental Engineering, 2001.

Gibson, W.M., The Physics of nuclear reactions, Pergamon Press, Oxford, 1980.

Glasstone, S. and Edlund, M.C., The elements of nuclear reactor theory, D. Van Nostrand Company, Princeton, 1957.

Greacen E. L., Soil water assessment by the neutron method, CSIRO Publication, East Melbourne, Victoria, Australia, 1981.

Haahr, V. and Olgaard, P.L., Comparative experimental and theoretical investigations of the neutronic method for measuring the water content in soil, Symposium on the Use of Isotopes and Radiation in Soil-Plant Nutrition Studies (IAEA), 129-147, 1965. 
Hughes, D.J. and Schwartz, R.B., Neutron Cross Sections BNL-325, Brookhaven National Laboratory, New York, 1958.

IAEA, Neutron moisture gauges: a guide-book on theory and practice, International Atomic Energy Agency, Vienna, 1970.

Iliffe, C. E., An introduction to nuclear reactor theory, Manchester University Press, Manchester, 1982.

Isbin, H., Introduction to nuclear reactor theory, Reinhold Publishing Corporation, New York, 1963

Morris, P.H. and William, D.J., Generalized calibration of a nuclear moisture/density depth gauge, ASTM Geotechnical Testing Journal, 13, 24-35, 1990.

Mughabghab, S.F., Divadeenam, M. and Holden, N.E., Neutron Cross Sections, Vol. 1, part A., Academic Press, New York, 1981.

Olgaard, P.L. and Haahr, V., Comparative experimental and theoretical investigations of the DM neutron moisture probe, Nuclear Engineering and Design, 5, 311-324, 1967.

Olgaard, P.L., On the theory of the neutronic method of measuring the water content in soil, Riso Report No. 97, Danish Atomic Energy Commission, 1965.

Rawls, W.J. and Asmussen, L.E, Neutron field calibration for soils in the Georgia coastal plain, Soil Science, 116 (4), 262-265, 1973.

Robinson, G.S and Harrington, B.V., AUS98 - the 1998 version of the AUS modular neutronics code system, ANSTO/E734, Australian Atomic Energy Commission, Research Establishment, Lucas Heights, N.S.W., 1998.

Schmugge, T.J., T.J. Jackson, and H.L. McKim, Survey of methods for soil moisture determination, water resource research, 16(16), 961-979, 1980. 
Silvestri, V., Sarkis, G., Bekkouche, N., Soulie, M., and Tabib, C., Laboratory and field calibration of a neutron depth moisture gauge for use in high water content soils, ASTM Geotechnical Testing Journal, 14 (1), 64-70, 1991.

Stacey, W. M., Nuclear reactor physics, John Wiley \& Sons, New York, 2001.

Stone, J.F., Neutron physics considerations in moisture probe design, Irrigation and Drainage, Proc. Nat. Conf. Am. Soc. Civil Engineers, Irrigation and Drainage Division, ASCE, 1-8, 1990.

Vachaud, G., Royer, J.M. and Cooper, J. D., Comparison of methods of calibration of a neutron probe by gravimetry or neutron-capture model, Journal of Hydrology, 34, 343-356, 1977.

Weinberg, A.M. and Wigner, E.P., The physical theory of neutron chain reactors, University of Chicago Press, Chicago, 1958.

Zweifel, P.F., Reactions physics, McGraw-Hill, New York, 1973. 